\title{
A High-Quality Draft Genome Sequence of Colletotrichum gloeosporioides sensu stricto SMCG1\#C, a Causal Agent of Anthracnose on Cunninghamia lanceolata in China
}

\author{
Lin Huang, ${ }^{1}$ Ki-Tae Kim, ${ }^{2}$ Ji-Yun Yang, ${ }^{1}$ Hyeunjeong Song, ${ }^{3}$ Gobong Choi, ${ }^{3}$ Jongbum Jeon, ${ }^{3}$ \\ Kyeongchae Cheong, ${ }^{3}$ Jaeho Ko, ${ }^{2}$ Haibin $\mathrm{Xu}^{4, \dagger}$ and Yong-Hwan Lee ${ }^{2,3,5, \dagger}$ \\ ${ }^{1}$ Co-Innovation Center for Sustainable Forestry in Southern China, College of Forestry, Nanjing Forestry \\ University, Nanjing, Jiangsu 210037, China; \\ ${ }^{2}$ Department of Agricultural Biotechnology, Seoul National University, Seoul 08826, Korea; \\ ${ }^{3}$ Interdisciplinary Program in Agricultural Genomics, Seoul National University; \\ ${ }^{4}$ College of Biology and the Environment, Nanjing Forestry University; \\ ${ }^{5}$ Center for Fungal Genetic Resources, Plant Genomics and Breeding Institute, and Research Institute of \\ Agriculture and Life Sciences, Seoul National University
}

\begin{abstract}
Colletotrichum has a broad host range and causes major yield losses of crops. The fungus Colletotrichum gloeosporioides is associated with anthracnose on Chinese fir. In this study, we present a high-quality draft genome sequence of $C$. gloeosporioides sensu stricto SMCG1\#C, providing a reference genomic data for further research on anthracnose of Chinese fir and other hosts.
\end{abstract}

Colletotrichum is one of the most important groups of phytopathogenic fungi in the world because of its scientific and economic importance (Dean et al. 2012). Colletotrichum gloeosporioides is a ubiquitous plant pathogen that infects a wide range of plant species (Weir et al. 2012). According to recent advances in taxonomy, C. gloeosporioides is considered a species complex and is segregated into 22 species and one subspecies (Weir et al. 2012). Chinese fir (Cunninghamia lanceolata) has been cultivated for over 3,000 years and contributes about $40 \%$ of timber in southern China (Huang et al. 2018; Shi et al. 2010). Anthracnose caused by $C$. gloeosporioides is one of the most serious fungal diseases on Chinese fir, which is widely distributed in the cultivated areas of Chinese fir, and causes enormous economic losses (Lan et al. 2015). C. gloeosporioides SMCG1\#C was isolated from the infected leaves of Chinese fir in Nanjing, China. Based on the phylogenetic tree calculated from the alignment of concatenated sequences of ITS, ACT, CAL, CHS-1, and GAPDH, strain SMCG1\#C was identified as C. gloeosporioides sensu stricto (unpublished data).

The genome of $C$. gloeosporioides SMCG1\#C was sequenced, using both PacBio Sequel System (Tianjin Biochip Corporation, Tianjin, China) and Illumina Hiseq X Ten System (Novogene Corporation, Beijing, China). A total of 519,294 reads and 171,464,766 pairedend 150-bp Illumina reads were generated, with respective coverages of $71 \times$ and $414 \times$. De novo assemblies were performed using Velvet version 1.2.10 (Zerbino and Birney 2008). We obtained 28 contigs with an average length of 2,210,112 bp, an N50 of 4,696,547 bp, and L50 of 5 . Finally, a draft genome of 18 scaffolds was produced by using BLASR and BLASTn algorithms (Camacho et al. 2009; Chaisson and Tesler 2012), a total of $61.9 \mathrm{Mb}, \mathrm{a} \mathrm{G}+\mathrm{C}$ content of $50.3 \%$, N50 of 5,209,244 bp, and L50 of 5 (Table 1). The validation of assembly

Lin Huang and Ki-Tae Kim contributed equally to this work.

${ }^{\dagger}$ Corresponding authors: H. Xu; E-mail: xuhaibin @ njfu.edu.cn and Y.-H. Lee; E-mail: yonglee@snu.ac.kr Accepted for publication 12 July 2018.

(c) 2019 The American Phytopathological Society
Funding:

This study was financially supported by the National Key R \& D Program of China (2017YFD0600102), the Major Project of Jiangsu Province University Natural Science Research (16KJA220002), and the Priority Academic Program Development of Jiangsu Higher Education Institutions (PAPD). This work was supported by grants from National Research Foundation of Korea (NRF2017R1A2A1A17069504, NRF2015M3A9B8028679). Ki-Tae is grateful for a graduate fellowship through the Brain Korea 21 Plus Program. 
Table 1. Genome assembly statistics of Colletotrichum gloeosporioides sensu stricto SMCG1\#C and the other $C$. gloeosporioides complex species

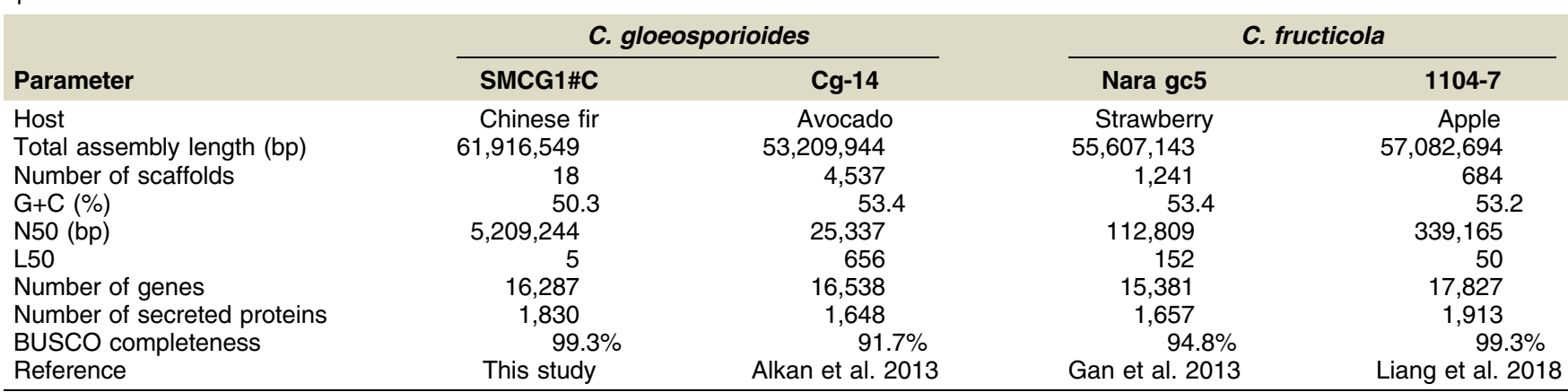

was achieved by BUSCO v3.0.2, using the fungi dataset (Waterhouse et al. 2018), and it showed $99.3 \%$ completeness for the assembled genome. A whole-genome alignment analysis using MUMmer v3.23 with species in the $C$. gloeosporioides species complex (Delcher et al. 2002), including C. gloeosporioides Cg-14, C. fructicola Nara gc5, and C. fructicola 1104-7 (Alkan et al. 2013; Gan et al. 2013; Liang et al. 2018), revealed that strain SMCG1\#C was close to the other C. gloeosporioides (83\% coverage) rather than the C. fructicola strains (69 and $71 \%$ coverage for Nara gc5 and 1104-7, respectively).

Structural annotation of the genome was performed using the MAKER v2.31.8 pipeline (Holt and Yandell 2011), and 16,287 protein-coding genes were identified. Among them, functions of 14,269 proteins ( $87.6 \%$ of proteome) were annotated by InterProScan 5.21-60.0 (Jones et al. 2014). According to the gene family pipelines previously described, 23 laccases, 48 peroxidases, 137 plant cell wall-degrading enzymes, 707 transcription factors, 281 Cytochrome P450, and 1,830 secretory protein-coding genes were predicted (Choi et al. 2010, 2013a, 2014; Park et al. 2008a and b). Among the secretome, 750 proteins were identified as small secreted proteins ( $<300$ amino acids) that might function as effectors (Kim et al. 2016). In addition, 1,076 CAZymes, 930 peptidases, and 246 lipases were predicted by dbCAN release 6.0, MEROPS release 12.0, and LED release 3.0 pipelines, respectively (Fischer and Pleiss 2003; Rawlings et al. 2018; Yin et al. 2012). The ortholog clustering analysis of $C$. gloeosporioides SMCG1\#C with the species complex and with $C$. orbiculare, C. graminicola, and C. higginsianum as outgroup (Dallery et al. 2017; Gan et al. 2013; O'Connell et al. 2012), using OrthoFinder v2.2.6 revealed 2,947 orthogroups specific to the species complex (Emms and Kelly 2015). Among them, 1,438 orthogroups were shared by all four strains and 547 orthogroups were only shared between the two $C$. gloeosporioides strains (557 genes in SMCG1\#C and 550 genes in Cg-14). Lastly, the strain SMCG1\#C had 407 orphan genes and 55 genes were functionally annotated as cation binding, transport, and integral component of membrane for the top three gene ontology terms.

The genome of $C$. gloeosporioides sensu stricto SMCG1\#C is, so far, the best quality genome within the published genomes of $C$. gloeosporioides species complex (Table 1), and it will be able to serve as a reference genome for comparative analysis of the species complex. The genome data has been deposited in the National Center for Biotechnology Information NCBI GenBank database under accession number QFRH00000000, PRJNA471237 for BioProject, and SAMN09205517 for BioSample. The genome sequence and gene models are also available from the Comparative Fungal Genomics Platform 2.0 (Choi et al. 2013b) and its sister databases described above.

\section{Literature Cited}

Alkan, N., Meng, X., Friedlander, G., Reuveni, E., Sukno, S., Sherman, A., Thon, M., Fluhr, R., and Prusky, D. 2013. Global aspects of pacC regulation of pathogenicity genes in Colletotrichum gloeosporioides as revealed by transcriptome analysis. Mol. Plant-Microbe Interact. 26:1345-1358.

Camacho, C., Coulouris, G., Avagyan, V., Ma, N., Papadopoulos, J., Bealer, K., and Madden, T. L. 2009. BLAST+: Architecture and applications. BMC Bioinformatics 10:421.
Chaisson, M. J., and Tesler, G. 2012. Mapping single molecule sequencing reads using basic local alignment with successive refinement (BLASR): Application and theory. BMC Bioinformatics 13:238.

Choi, J., Cheong, K., Jung, K., Jeon, J., Lee, G. W., Kang, S., Kim, S., Lee, Y. W., and Lee, Y. H. 2013b. CFGP 2.0: A versatile web-based platform for supporting comparative and evolutionary genomics of fungi and Oomycetes. Nucleic Acids Res. 41 (D1):D714-D719. 
Choi, J., Détry, N., Kim, K. T., Asiegbu, F. O., Valkonen, J. P. T., and Lee, Y. H. 2014. fPoxDB: Fungal peroxidase database for comparative genomics. BMC Microbiol. 14:117.

Choi, J., Kim, K. T., Jeon, J., and Lee, Y. H. 2013a. Fungal plant cell wall-degrading enzyme database: A platform for comparative and evolutionary genomics in fungi and Oomycetes. BMC Genomics 14 (Suppl 5):S7.

Choi, J., Park, J., Kim, D., Jung, K., Kang, S., and Lee, Y. H. 2010. Fungal secretome database: Integrated platform for annotation of fungal secretomes. BMC Genomics 11:105.

Dallery, J. F., Lapalu, N., Zampounis, A., Pigné, S., Luyten, I., Amselem, J., Wittenberg, A. H. J., Zhou, S., de Queiroz, M. V., Robin, G. P., Auger, A., Hainaut, M., Henrissat, B., Kim, K. T., Lee, Y. H., Lespinet, O., Schwartz, D. C., Thon, M. R., and O'Connell, R. J. 2017. Gapless genome assembly of Colletotrichum higginsianum reveals chromosome structure and association of transposable elements with secondary metabolite gene clusters. BMC Genomics 18:667.

Dean, R., Van Kan, J. A. L., Pretorius, Z. A., Hammond-Kosack, K. E., Di Pietro, A., Spanu, P. D., Rudd, J. J., Dickman, M., Kahmann, R., Ellis, J., and Foster, G. D. 2012. The top 10 fungal pathogens in molecular plant pathology. Mol. Plant Pathol. 13:414-430.

Delcher, A. L., Phillippy, A., Carlton, J., and Salzberg, S. L. 2002. Fast algorithms for large-scale genome alignment and comparison. Nucleic Acids Res. 30:2478-2483.

Emms, D. M., and Kelly, S. 2015. OrthoFinder: Solving fundamental biases in whole genome comparisons dramatically improves orthogroup inference accuracy. Genome Biol. 16:157.

Fischer, M., and Pleiss, J. 2003. The Lipase Engineering Database: A navigation and analysis tool for protein families. Nucleic Acids Res. 31:319-321.

Gan, P., Ikeda, K., Irieda, H., Narusaka, M., O'Connell, R. J., Narusaka, Y., Takano, Y., Kubo, Y., and Shirasu, K. 2013. Comparative genomic and transcriptomic analyses reveal the hemibiotrophic stage shift of Colletotrichum fungi. New Phytol. 197:1236-1249.

Holt, C., and Yandell, M. 2011. MAKER2: An annotation pipeline and genomedatabase management tool for second-generation genome projects. BMC Bioinformatics 12:491.

Huang, L., Zhu, Y. N., Yang, J. Y., Li, D.-W., Li, Y., Bian, L. M., and Ye, J. R. 2018. Shoot blight on Chinese fir (Cunninghamia lanceolata) is caused by Bipolaris oryzae. Plant Dis. 102:500-506.

Jones, P., Binns, D., Chang, H. Y., Fraser, M., Li, W., McAnulla, C., McWilliam, H., Maslen, J., Mitchell, A., Nuka, G., Pesseat, S., Quinn, A. F., Sangrador-Vegas, A., Scheremetjew, M., Yong, S. Y., Lopez, R., and Hunter, S. 2014. InterProScan 5: Genome-scale protein function classification. Bioinformatics 30:1236-1240.

Kim, K. T., Jeon, J., Choi, J., Cheong, K., Song, H., Choi, G., Kang, S., and Lee, Y. H. 2016. Kingdom-wide analysis of fungal small secreted proteins (SSPs) reveals their potential role in host association. Front. Plant Sci. 7:186.

Lan, X., Dong, L., Huang, K., Chen, D., Li, D., and Mo, L. 2015. Main species and prevention research on diseases and pests of Cunninghamia lanceolata. Guangxi For. Sci. 44:162-167.
Liang, X., Wang, B., Dong, Q., Li, L., Rollins, J. A., Zhang, R., and Sun, G. 2018. Pathogenic adaptations of Colletotrichum fungi revealed by genome wide gene family evolutionary analyses. PLoS One 13:e0196303.

O'Connell, R. J., Thon, M. R., Hacquard, S., Amyotte, S. G., Kleemann, J., Torres, M. F., Damm, U., Buiate, E. A., Epstein, L., Alkan, N., Altmüller, J., AlvaradoBalderrama, L., Bauser, C. A., Becker, C., Birren, B. W., Chen, Z., Choi, J., Crouch, J. A., Duvick, J. P., Farman, M. A., Gan, P., Heiman, D., Henrissat, B., Howard, R. J., Kabbage, M., Koch, C., Kracher, B., Kubo, Y., Law, A. D., Lebrun, M. H., Lee, Y. H., Miyara, I., Moore, N., Neumann, U., Nordström, K., Panaccione, D. G., Panstruga, R., Place, M., Proctor, R. H., Prusky, D., Rech, G., Reinhardt, R., Rollins, J. A., Rounsley, S., Schardl, C. L., Schwartz, D. C., Shenoy, N., Shirasu, K., Sikhakolli, U. R., Stüber, K., Sukno, S. A., Sweigard, J. A., Takano, Y., Takahara, H., Trail, F., van der Does, H. C., Voll, L. M., Will, I., Young, S., Zeng, Q., Zhang, J., Zhou, S., Dickman, M. B., Schulze-Lefert, P., Ver Loren van Themaat, E., Ma, L. J., and Vaillancourt, L. J. 2012. Lifestyle transitions in plant pathogenic Colletotrichum fungi deciphered by genome and transcriptome analyses. Nat. Genet. 44:1060-1065.

Park, J., Lee, S., Choi, J., Ahn, K., Park, B., Park, J., Kang, S., and Lee, Y. H. 2008a. Fungal cytochrome P450 database. BMC Genomics 9:402.

Park, J., Park, J., Jang, S., Kim, S., Kong, S., Choi, J., Ahn, K., Kim, J., Lee, S., Kim, S., Park, B., Jung, K., Kim, S., Kang, S., and Lee, Y. H. 2008b. FTFD: An informatics pipeline supporting phylogenomic analysis of fungal transcription factors. Bioinformatics 24:1024-1025.

Rawlings, N. D., Barrett, A. J., Thomas, P. D., Huang, X., Bateman, A., and Finn, R. D. 2018. The MEROPS database of proteolytic enzymes, their substrates and inhibitors in 2017 and a comparison with peptidases in the PANTHER database. Nucleic Acids Res. 46 (D1):D624-D632.

Shi, J., Zhen, Y., and Zheng, R. H. 2010. Proteome profiling of early seed development in Cunninghamia lanceolata (Lamb.) Hook. J. Exp. Bot. 61: 2367-2381.

Waterhouse, R. M., Seppey, M., Simão, F. A., Manni, M., loannidis, P., Klioutchnikov, G., Kriventseva, E. V., and Zdobnov, E. M. 2018. BUSCO applications from quality assessments to gene prediction and phylogenomics. Mol. Biol. Evol. 35: 543-548.

Weir, B. S., Johnston, P. R., and Damm, U. 2012. The Colletotrichum gloeosporioides species complex. Stud. Mycol. 73:115-180.

Yin, Y., Mao, X., Yang, J., Chen, X., Mao, F., and Xu, Y. 2012. dbCAN: A web resource for automated carbohydrate-active enzyme annotation. Nucleic Acids Res. 40:W445-W451.

Zerbino, D. R., and Birney, E. 2008. Velvet: Algorithms for de novo short read assembly using de Bruijn graphs. Genome Res. 18:821-829.

Author-Recommended Internet Resource

Comparative Fungal Genomics Platform: http://cfgp.riceblast.snu.ac.kr 